\title{
Conceito da Comunicação na formação dos fonoaudiólogos no ensino público do Rio Grande do Sul
}

\author{
Cibele Gulartt Avendano \\ Mestranda do Programa de Pós-graduação em Educação em Ciências - UFRGS

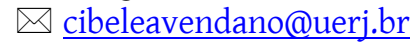 \\ Tiago Pereira de Souza \\ Doutorando do Programa de Pós-graduação em Educação em Ciências - UFRGS \\ Erissandra Gomes \\ Docente do Programa de Pós-graduação em Educação em Ciências - UFRGS \\ Sidney Gonçalves Vieira \\ Pós-Doutor pelo Departamento de Geografia da Universidade de Barcelona
}

Recebido em 15 de setembro de 2020

Aceito em 6 de julho de 2021

\begin{abstract}
Resumo:
A área da saúde passa por constantes transformações no seu modelo de atenção ao cuidado. A comunicação está fortemente interligada a estes processos, potencializando e contribuindo com o aprimoramento das relações estabelecidas, tanto pelo mundo do trabalho na saúde, quanto na formação de profissionais da área. Este estudo tem como objeto a formação do profissional Fonoaudiólogo, oriundo de três Instituições de Ensino Superior (IESs) Federais do Rio Grande Sul, apresentadas como: IES A, IES B e IES C. Objetiva verificar a existência do conceito e do significado atribuído à comunicação nos documentos norteadores do ensino da Fonoaudiologia por meio de uma análise de conteúdo nas Diretrizes Curriculares Nacionais (DCNs) e no Perfil do Egresso (PE) contido nos Projetos Pedagógicos Curriculares (PPCs). O estudo apresentou a frequência do vocábulo "comunicação" em cada documento estudado (DCN n=19; PE n=5) correlacionando os termos com os trechos elucidados no texto. A classificação polissêmica encontrada indicou seis aplicações distintas do termo no corpus da IES A e da IES B: presente em títulos e tópicos, atribuído à expressão "comunicação humana", associado à expressão "tecnologias de comunicação e informação", vinculado à expressão "comunicação verbal e não verbal", associado a habilidades de liderança, relacionados a comunicação com pacientes, responsáveis e profissionais de outras áreas. Verificou-se a ausência do termo no PE da IES C. Foi possível também perceber discrepâncias conceituais nas DCNs, no que diz respeito ao entendimento da comunicação enquanto habilidade e não como uma competência profissional.
\end{abstract}

Palavras-chave: Comunicação, Fonoaudiologia, Ensino superior, Avaliação educacional.

\section{Concept of Communication in the training of speech therapists in public education in Rio Grande do Sul}

\begin{abstract}
:
The health area is undergoing constant changes in its model of care. Communication is strongly interconnected to these processes, enhancing and contributing to the improvement of the relationships established, both in the world of health work and in the training of professionals in the area. This study has as its object the formation of the professional Speech-Language Pathologist, from three Federal Higher Education Institutions (HEIs) of Rio Grande Sul, presented as: HEI A, HEI B and
\end{abstract}


HEI C. It aims to verify the existence of the concept and the meaning attributed to the communication in the guiding documents of Speech Therapy teaching through a content analysis in the National Curriculum Guidelines (DCNs) and in the Graduate Profile (PE) contained in the Curricular Pedagogical Projects (PPCs). The study presented the frequency of the word "communication" in each document studied ( $D C N$ n=19; PE n=5) correlating the terms with the excerpts elucidated in the text. The polysemic classification found indicated six different applications of the term in the corpus of HEI A and HEI B: present in titles and topics, attributed to the expression "human communication", associated with the expression "communication and information technologies", linked to the expression "verbal communication" and non-verbal", associated with leadership skills, related to communication with patients, guardians and professionals from other areas. There was an absence of the term in the PE of IES C. It was also possible to notice conceptual discrepancies in the DCNs, with regard to the understanding of communication as a skill and not as a professional competence.

Keywords: Communication, Speech therapy, Higher education, Educational evaluation.

\section{Concepto de Comunicación em la Formación de Terapeuta del Habla em Educación Pública em Rio Grande do Sul}

\section{Resumen:}

El área de la salud está experimentando constantes cambios en su modelo de atención. La comunicación está fuertemente interconectada con estos procesos, potenciando y contribuyendo a la mejora de las relaciones que se establecen, tanto en el mundo del trabajo sanitario como en la formación de los profesionales del área. Este estudio tiene como objeto la formación del Profesional Patólogo del Habla y el Lenguaje, de tres Instituciones Federales de Educación Superior (IES) de Rio Grande Sul, presentado como: IES A, IES B y IES C. Tiene como objetivo verificar la existencia de la concepto y el significado atribuido a la comunicación en los documentos rectores de la docencia de Logopedia a través de un análisis de contenido en las Directrices Curriculares Nacionales (DCNs) y en el Perfil de Posgrado (PE) contenido en los Proyectos Pedagógicos Curriculares (PPCs). El estudio presentó la frecuencia de la palabra "comunicación" en cada documento estudiado (DCN n=19; PE n=5) correlacionando los términos con los extractos aclarados en el texto. La clasificación polisémica encontrada indicó seis aplicaciones diferentes del término en el corpus de las IES A y IES B: presente en títulos y temas, atribuido a la expresión "comunicación humana", asociada a la expresión "tecnologías de la comunicación y la información", vinculada a la expresión "comunicación verbal" y no verbal", asociada a las habilidades de liderazgo, relacionada con la comunicación con pacientes, tutores y profesionales de otras áreas. Hubo ausencia del término en el PE de IES C. También se pudieron notar discrepancias conceptuales en el DCN, en lo que respecta a la comprensión de la comunicación como habilidad y no como competencia profesional.

Palabras clave: Comunicación, Terapia del lenguaje, Enseñanza superior, Evaluación educativa.

\section{INTRODUÇÃO}

A comunicação é inerente aos humanos (BRAGA; CALAZANS, 2001), compreendida como prática advinda da interação, expressa por aspectos verbais, escrita, gestos, distância entre os participantes e aspectos não verbais (FERMINO; CARVALHO, 2007). Na área da saúde, a comunicação é um componente que sempre esteve presente e se modificou juntamente com os modelos assistenciais propostos. Inicialmente entendida somente como uma transmissão de mensagens do emissor para o receptor de forma unilinear impositiva (TEIXEIRA, 1997), 
como visto no início do século XX, quando era atrelada a ações fiscalizatórias, a exemplo da Revolta das Vacinas de 1904.

Para transcender a este modelo arcaico e policialesco, transformações conceituais, políticas, éticas, educacionais e sociais na área da saúde aconteceram em relação à comunicação. Com o passar dos anos, a aplicação dos pressupostos comunicacionais na saúde evoluiu para a esfera de política pública, hoje não mais impositiva e sim educativa, nos termos da Constituição Brasileira, adequadamente caracterizada como serviço público de interesse econômico e político geral (TABORDA, 2017).

Cabe destacar que, embora reconhecida fundamentalmente por sua importância na consolidação do cuidado em saúde, a comunicação social ainda não conseguiu questionar algumas práticas no ensino e no trabalho, enraizadas no modelo tradicional, mecanicista e predominantemente voltado para a transmissão de informações que impõem comportamentos aos sujeitos (PEREIRA, A. L. F., 2003). Quando o modelo de atenção desconsidera a comunicação enquanto competência profissional, reproduz um trabalho essencialmente fragmentado e focado na doença, pois não inclui o outro no processo dialógico, que faz com que o cuidado seja autoritário e coercitivo. Por outro lado, a comunicação modifica conhecimento e atitudes, visando a busca de comportamentos favoráveis à saúde (ROCHA, 2003).

No universo acadêmico, mais especificamente na produção do conhecimento na área da saúde, adquire novos significados quando objetiva a contribuição no aprimoramento do processo de trabalho com o viés para a socialização do conhecimento e na busca da solução de problemáticas específicas de cada população (BURGOS et al., 2015). Quando a comunicação social é compreendida como uma aliada ao processo de ensino nas formações, amplia a cooperação e aproximação entre os pesquisadores, colabora para o reconhecimento das pesquisas e revalida as competências e a credibilidade do pesquisador, além de possibilitar um acesso mais contemporâneo, polissêmico e atrativo, tanto nas pesquisas quanto nos projetos de extensão (BURGOS et al., 2015; DROESCHER; SILVA, 2018; RODRIGUES; BLATTMANN, 2014).

Além disso, a relação entre o profissional de saúde e seu paciente, cliente ou usuário também sofre interferência direta no que diz respeito ao seu processo terapêutico quando consideramos a comunicação como determinante (CAPRARA; FRANCO, 1999). Este aspecto 
vem sendo considerado em alguns espaços de formação em outros países, que reconhecem a importância de incorporar o conteúdo em seu currículo, embora enfrentando dificuldades na contratação de docentes capacitados (AGUIAR et al., 2014; DONATO; GOMES, 2010; NESTEL et al., 2002; ROSSI; BATISTA, 2006).

A formação em saúde no Brasil é norteada pelas Diretrizes Curriculares Nacionais (DCNs) e pelas diretrizes do Sistema Único de Saúde (SUS). A partir dessas configurações, temos referências que estabelecem o perfil profissional a ser formado; as oportunidades e os fatores de exposição à aprendizagem; os Projetos Pedagógicos Curriculares (PPCs); a organização e orientação dos currículos e das práticas; produção do conhecimento e as relações entre ensino e serviço (CECCIM; FEUERWERKER, 2004). O Conselho Nacional de Educação aprovou em 2001 as DCNs dos Cursos de graduação da Área da Saúde, no intuito de garantir uma formação básica sólida, capaz de preparar o egresso para enfrentar os desafios das transformações da sociedade, do mercado de trabalho e das condições contemporâneas do exercício profissional (GARCIA, 2015).

A promulgação das DCNs da Fonoaudiologia, em 2002, destacou a necessidade de maior articulação entre teoria e prática; a integração entre instituições de ensino e sistema de saúde e a diversificação dos cenários de aprendizagem. A comunicação foi contemplada no documento, sendo atribuída ao contato com outros profissionais, confidencialidade, comunicação verbal e não verbal, leitura, escrita enquanto habilidades, além do domínio de, no mínimo, uma língua estrangeira e de tecnologias de comunicação e informação (LEMOS, 2012).

Neste sentido a formação em Fonoaudiologia, inserida no rol de profissões da área da saúde, foi o objeto de estudo desta pesquisa, que objetivou verificar a existência do conceito e do significado atribuído no que diz respeito à comunicação nos documentos norteadores de sua formação. Delimitou-se a relação entre a formação do profissional Fonoaudiólogo e a comunicação, a partir de uma análise documental das DCNs e dos Perfis dos Egressos (PEs) encontrados nos PPCs elaborados pelas Instituições de Ensino Superior (IESs) Federais no Rio Grande do Sul (RS). 


\section{METODOLOGIA}

Este artigo se trata de uma pesquisa de caráter quali-quantitativo, apresentando uma análise de conteúdo das DCNs dos cursos de graduação de Fonoaudiologia e do PE, descritos nos PPCs dos cursos de Fonoaudiologia nas IESs Federais do RS, aqui representadas e denominadas ao longo do estudo por IES A, IES B e IES C, utilizando tal análise como procedimento para investigar suas interfaces (CÂMARA, 2013) e valendo-se também da estatística de análise textual (CAMARGO; JUSTO, 2013).

Configura-se como um estudo de natureza exploratória e descritiva, estabelecendo correlações para identificar a existência e aplicação dos conceitos de comunicação social nas DCNs e nos PPCs da graduação de Fonoaudiologia das instituições estudadas.

Primeiramente, os PPC foram pesquisados tendo como fonte principal os sites das IESs, de onde foram extraídos os PEs. As DCNs do Curso de Fonoaudiologia foram encontradas no site do portal do Ministério da Educação (BRASIL, 2002). Foram construídos gráficos de análise textual e frequência de termos a partir do software IRAMUTEQ, utilizando as ferramentas para aplicação do cálculo de frequência de palavras (estatística); nuvem de palavras e análise de similitude. A escolha de tais processos foi estabelecida pela compatibilidade com os dados apresentados ao programa e, por demonstrarem eficiência e eficácia no processamento dos mesmos, para posterior inferência de significados aos gráficos (CAMARGO; JUSTO, 2013).

A fim de alcançar os objetivos propostos inicialmente, o texto das DCNs foi salvo em extensão “.txt”, por ser o formato padrão exigido pelo IRAMUTEQ. Posteriormente, aglutinamos os PEs em um único arquivo, salvando-o também no padrão do programa de análise. Nesta etapa, o conteúdo dos campos de metadados de cada PE foi separado por um padrão de caracteres (*** *PE_IES_A, *** *PE_IES_B e ****PE_IES_C), onde “A, B e C" correspondem a um inteiro, permitindo seu processamento de forma unificada. Deste modo, dois corpus textuais formaram a base de dados, salvos com codificação UTF-8 no software Bloco de Notas (DCN e PE). 


\section{RESULTADOS E DISCUSSÃo}

$\mathrm{Na}$ análise do corpus DCN foram observadas 2.087 ocorrências de palavras, sendo 606 formas distintas. O corpus do texto do PE das três IESs (A, B e C) apresentou 2.895 palavras e 863 formas diferentes.

Para facilitar o entendimento dos resultados e sistematizar a discussão, organizamos inicialmente a partir da apresentação da análise por meio de nuvens de palavras seguida de sua discussão e posteriormente a análise de conectividade e similitude das DCNs e PEs respectivamente.

\section{Comunicação nas nuvens}

Inicialmente, optou-se pelo gráfico de nuvem de palavras, que agrupa as palavras e as organiza em função da sua frequência, como ilustram as Figuras 1 e 2, considerando-se a análise geral de termos, para facilitar a visualização dos vocábulos mais utilizados nos corpus.

A amostra foi delimitada em uma variação de frequência (mínima e máxima) de palavras entre 5 e 23 para as DCNs e 3 a 37 para os PEs. Estas referências foram estabelecidas para que o termo "comunicação" pudesse aparecer nas duas imagens. No corpus das DCNs, o vocábulo comunicação possui $(n=5)$; ou seja, a frequência mínima para que o programa pudesse captar o vocábulo foi 5. A variação em ambos os corpus foi escolhida para não comprometer a qualidade na visualização da imagem e considerando a significância semântica dos vocábulos.

A análise estatística, feita pelo programa e visualizada nas nuvens de palavras, mostra uma maior prevalência das palavras "fonoaudiologia" (n=23), "curso" ( $n=21)$ e "saúde" (n=18) no corpus das DCNs na Figura 1 e "saúde" (n=37), "formação" (n=25) e "comunicação" (n=19). Passamos para a análise do vocábulo "comunicação", um dos objetos principais deste estudo. 
Figura 1. Nuvem de Palavras das Diretrizes Curriculares Nacionais da graduação de Fonoaudiologia

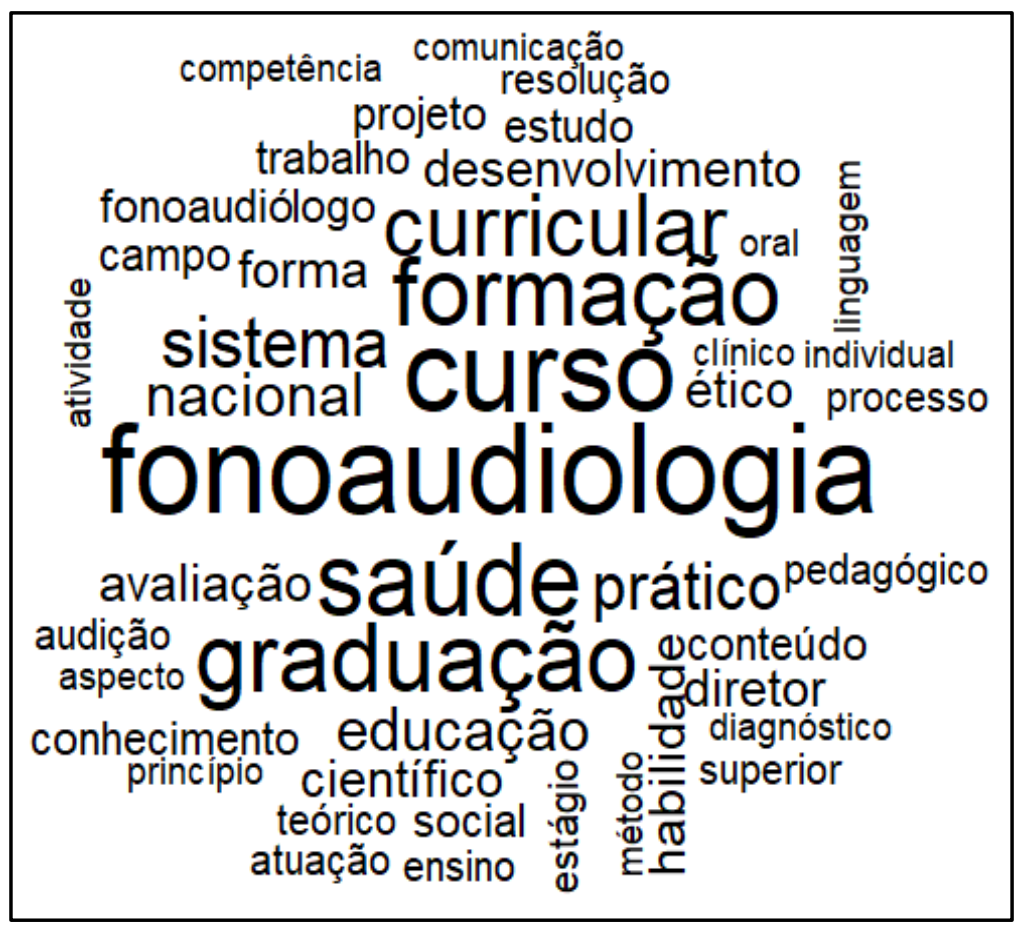

Fonte: Elaborada pelos autores (2020).

Na Figura 1, "comunicação" aparece contida na parte centro-superior na imagem de forma discreta $(n=5)$, sendo que uma delas aparece no texto como título e quatro têm sentido textual, conforme veremos a seguir: “[...] a comunicação envolve comunicação verbal, não verbal e habilidades de escrita e leitura [...]" (BRASIL, 2002, n. p., grifo nosso).

O trecho acima fala sobre comunicação verbal e não verbal, porém não traz com clareza a que se referem os termos. Caberia a reflexão mais aprofundada de que neste contexto faz-se necessária uma adequação terminológica, para que o profissional entenda que uma comunicação não deve se estabelecer de maneira puramente técnica, mas também através de uma abordagem expressiva do cuidar, cuidado este que se dá também pela comunicação, seja ela verbal ou não-verbal (FERREIRA, 2006).

Outro trecho em destaque trata sobre tecnologias de comunicação: “[...] O domínio de pelo menos uma língua estrangeira e de tecnologias de comunicação e informação [...]" (BRASIL, 2002, n. p., grifo nosso). Mais uma vez, não é devidamente explicitada a definição desta terminologia. Tecnologias de comunicação e informação não se resumem apenas a 
aplicativos de transmissão de mensagens de textos, imagens ou áudios, mas sim à base material para a expansão de redes em toda a estrutura sociocultural da sociedade moderna, além da democratização da informação (FREY, 2003; PEREIRA, D. M.; SILVA, 2011).

Destaca-se também o seguinte trecho: “[...] liderança envolve compromisso, responsabilidade, empatia, habilidade para tomada de decisões, comunicação e gerenciamento de forma efetiva e eficaz [...]" (BRASIL, 2002, n. p., grifo nosso). Nesse trecho, o termo se relaciona a habilidades de liderança, estando associado a outros conceitos não relacionados diretamente à comunicação social, mas sim a relações interpessoais estudadas principalmente nas áreas de administração (COSTA; BENTO, 2015; PINHEIRO et al., 2020). Esse trecho também será abordado mais adiante na análise de similitude.

Na Figura 2, a palavra "comunicação" aparece com destaque, de forma central e com tamanho evidente, porém é necessário fazer a análise de forma global (corpus como um todo) e individual (IES A, IES B e IES C).

Figura 2. Nuvem de Palavras do Perfil do Egresso das Instituições estudadas

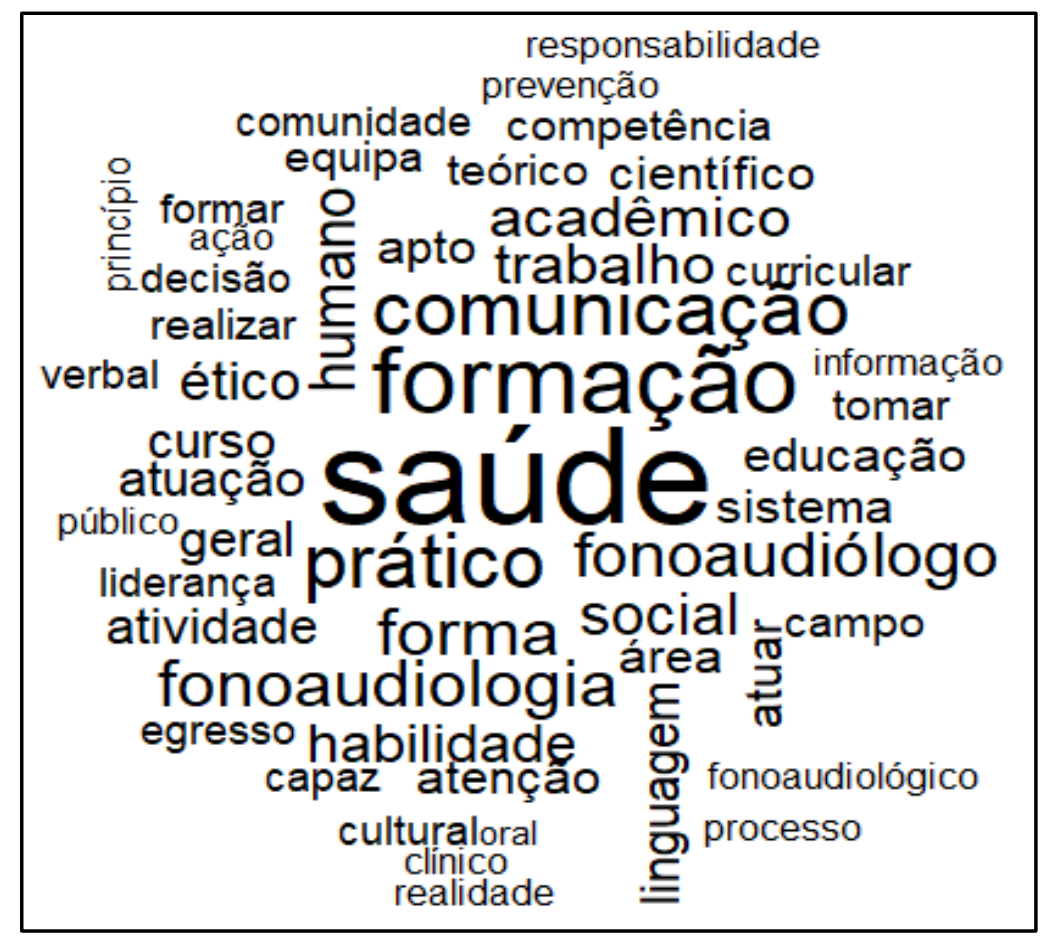

Fonte: Elaborada pelos autores (2020). 
De forma geral, o corpus dos PEs demonstra a frequência da palavra "comunicação" ( $n=19)$, sendo uma das palavras mais recorrentes do texto, o que, em uma análise superficial, levaria a crer que a representatividade semântica seria alta. Porém, ao analisarmos por IES, temos: IES A ( $n=6)$, IES B ( $n=13)$, IES C ( $n=0)$, conforme distribuição polissêmica do termo.

A IES A, apresenta o PE com o vocábulo "comunicação" repetido 6 vezes, sendo: 1 no título, 1 termo associado à expressão "comunicação humana", 1 no termo associado à expressão "tecnologias de comunicação e informação" (FREY, 2003; PEREIRA, D. M.; SILVA, 2011), 2 nos termos associados à expressão "comunicação verbal e não verbal" (FERREIRA, 2006) e 1 no termo associado a habilidades de liderança (COSTA; BENTO, 2015; PINHEIRO et al., 2020).

Por sua vez, a IES B demonstrou a maior frequência do termo estudado (n=13). A caracterização do termo dentro do corpus fica disposta da seguinte forma: 1 no título, 5 vezes associado à expressão "comunicação humana"; 1 no termo relacionado à expressão “comunicação verbal e não verbal” (FERREIRA, 2006), 1 no relativo à expressão "tecnologias de comunicação e informação" (FREY, 2003; PEREIRA, D. M.; SILVA, 2011), 2 nos termos relacionados a habilidades de liderança (COSTA; BENTO, 2015; PINHEIRO et al., 2020) e 3 nos termos relacionados à comunicação com pacientes, responsáveis e profissionais de outras áreas, sendo este último discutido na sequência.

A IES B foi a única que elencou a preocupação em destacar no PE a relação comunicacional com pacientes, familiares e outros profissionais, demonstrando reconhecer a comunicação social como instrumento indispensável para a articulação do cuidado e do acolhimento adequado das demandas dos pacientes e de seus familiares. O vínculo, a satisfação, as relações de confiança, o entendimento da doença e do processo de tratamento possibilitam, além de uma maior adesão, a corresponsabilização no processo saúde-doença (BOISSY et al., 2016; MULLAN; KOTHE, 2010; RANJAN; KUMARI; CHAKRAWARTY, 2015).

O software não identificou a presença do termo "comunicação" no PE da IES C, independentemente de sua polissemia, contrariando a recomendação de pesquisas no sentido de incluir a temática em sua formação (BACHMANN et al., 2013; ELLIS; DIETZ, 2017; KITSON et al., 2013; KURTZ; COOKE, 2017). 
A relação entre os vocábulos que formam a expressão "comunicação humana" se estabelece no corpus em 6 encontros, sendo que destes 1 está na IES A e 5 estão na IES B. Esta configuração se relaciona com a definição do objeto de estudo da profissão fonoaudiólogo, que trata dos distúrbios da comunicação humana (FERIGOLLO; KESSLER, 2017).

Para aprofundarmos estas configurações, ocorrências e estabelecemos as conexões do vocábulo "comunicação" dentro dos corpus das DCNs e dos PEs, a fim de compreendermos a configuração do texto, a estrutura da representação textual e fazermos inferências, passamos a utilizar a análise de similitudes como disparadora da discussão (MARCHAND; RATINAUD, 2011).

\section{Comunicação e suas conexões}

Foram utilizados para essa amostra 39 vocábulos com índice de frequência e relevância temática, para os quais a frequência variou de 3 até 23 nas DCNs e 25 vocábulos com índice de frequência que variou de 5 a 32 na análise de similitude dos PEs das IESs. A escolha do número de palavras levou em consideração aspectos relacionados à melhor visualização e vocábulos mais significativos. Destacamos que, para realizar essa análise, foram excluídas as seguintes classes gramaticais: advérbios, advérbios suplementares, conjunções, preposições, verbos e verbos suplementares, por não possuírem valor semântico.

As Figuras 3 e 4 apontam para as palavras "fonoaudiologia" como vértice central nas DCNs e "saúde" e "formação" nos PEs. Este resultado indica as DCNs, enquanto documento norteador da formação em Fonoaudiologia no Brasil, sendo, portanto, de suma importância que a construção dos PPCs, mais especificamente que a descrição dos PEs, tenham consonância com as DCNs (FRANCO; CUBAS; FRANCO, 2014). 
Figura 3.Análise de Proximidade e Similitude das Diretrizes Curriculares Nacionais da graduação de Fonoaudiologia

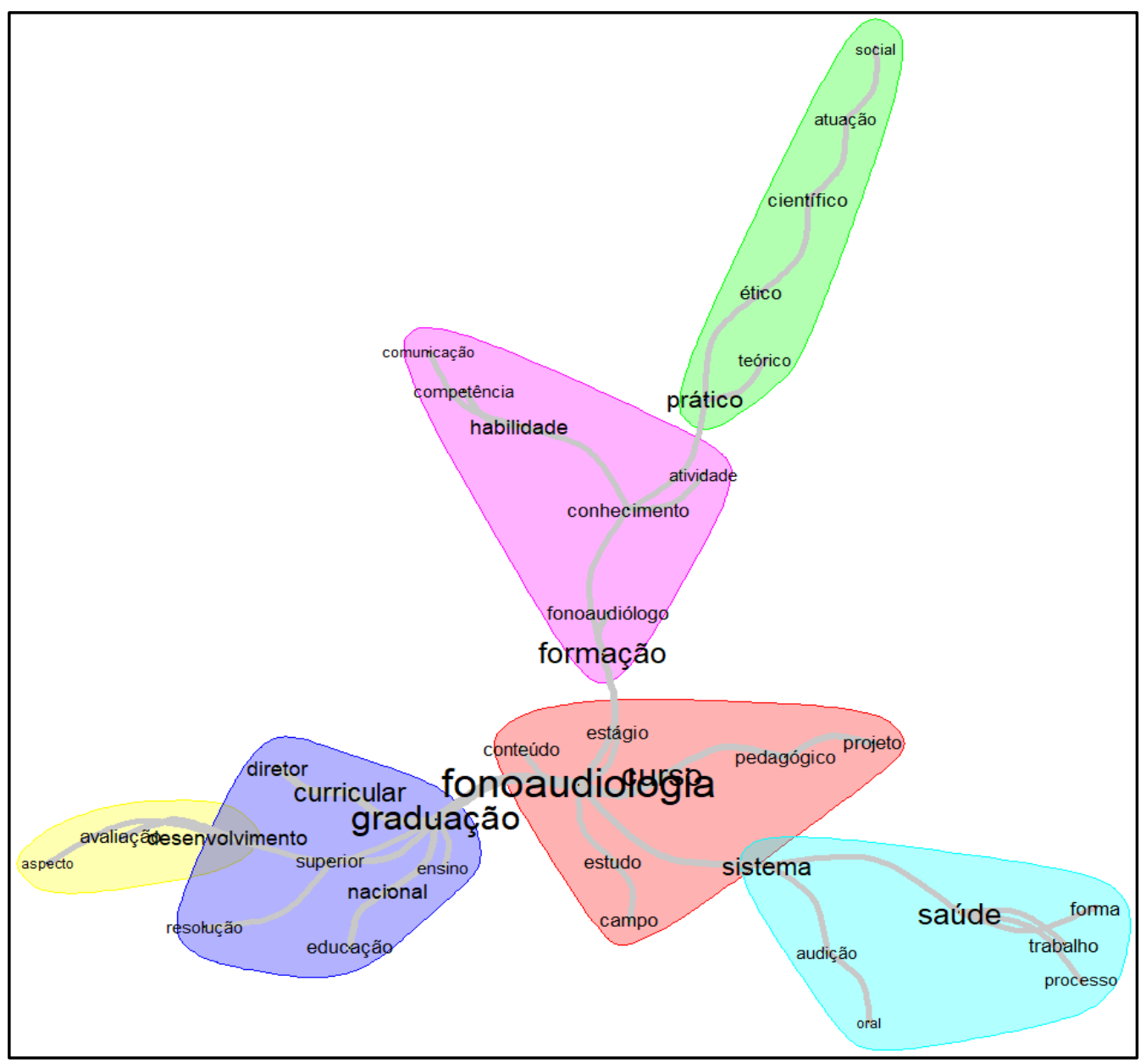

Fonte: Elaborada pelos autores (2020).

As DCNs, representadas na Figura 3, são articuladas a partir de um vértice central denominado de "fonoaudiologia", de onde partem três ramificações principais. São elas:

a) graduação: se aproximando do "ensino" "superior" e sua oferta "curricular";

b) sistema:

- estudos do corpo "audição" e "oral”;

- "sistema" de "saúde" enquanto "forma" e "processos" de "trabalho";

c) formação do Fonoaudiólogo:

- "conhecimentos", "habilidades" e "competências", de modo que, no texto, a

"comunicação" está associada à "habilidade";

- "prática embasada na "ética", "teórica", "científica" e "atuação social”.

A análise de similitude das DCNs da formação dos Fonoaudiólogos mostra que o egresso do curso deve ter a habilidade de comunicação, ou seja, o documento norteador da 
formação dos profissionais da área que trata dos distúrbios da comunicação humana, reduz a "comunicação" a uma habilidade e não uma competência profissional (FLEURY; FLEURY, 2001; SAUPE, 2006).

Esta reflexão é importante e exige o entendimento mais aprofundado de alguns conceitos básicos que formam a tríade desta competência profissional, composta pela interrelação, primeiramente por conhecimentos obtidos por meio de leituras, que possibilitam ao profissional o domínio do saber e a capacidade de tomar decisões para solucionar problemas, e posteriormente por habilidades advindas de experiências obtidas por repetições, que fornecem ao profissional o domínio do saber fazer e a capacidade de tomar decisões e solucionar problemas. E, por fim, atitudes adquiridas pela observação que confere ao profissional o domínio ético e afetivo de um saber ser e saber conviver (FLEURY; FLEURY, 2001; SAUPE, 2006).

Partindo da concepção apresentada anteriormente, é possível inferir que a proposição de formação de egresso hábil em comunicação social, apresentada pelas DCNs, dificilmente poderá ser atingida na prática, uma vez que é legítimo o seu foco voltado para as repetições em busca de habilidades, sem o domínio do conhecimento técnico em comunicação social, não possibilitando a real transformação em atitudes. O desenvolvimento da competência profissional vincula-se não apenas à aquisição de conhecimentos por métodos tradicionais, mas também a processos de aprendizagem informais que ocorrem em momentos e espaços distintos (BOMFIM, 2012).

$\mathrm{Na}$ análise de similitude do PE, como ilustra a Figura 4, dois vocábulos originam a estrutura do corpus. A palavra "saúde" estabelece de forma mais significativa (semanticamente e visualmente) a "forma" de "atuação", o "trabalho" enquanto "tomada" de “decisões” e, de modo "geral”, suas "competências" e "habilidades". Chamamos atenção para o fato de que, no PE, o vocábulo "comunicação" não está vinculado a estes conceitos, diferentemente das DNC, como vimos anteriormente. 
Figura 4.Análise de Proximidade e Similitude do Perfil do Egresso das Instituições estudadas

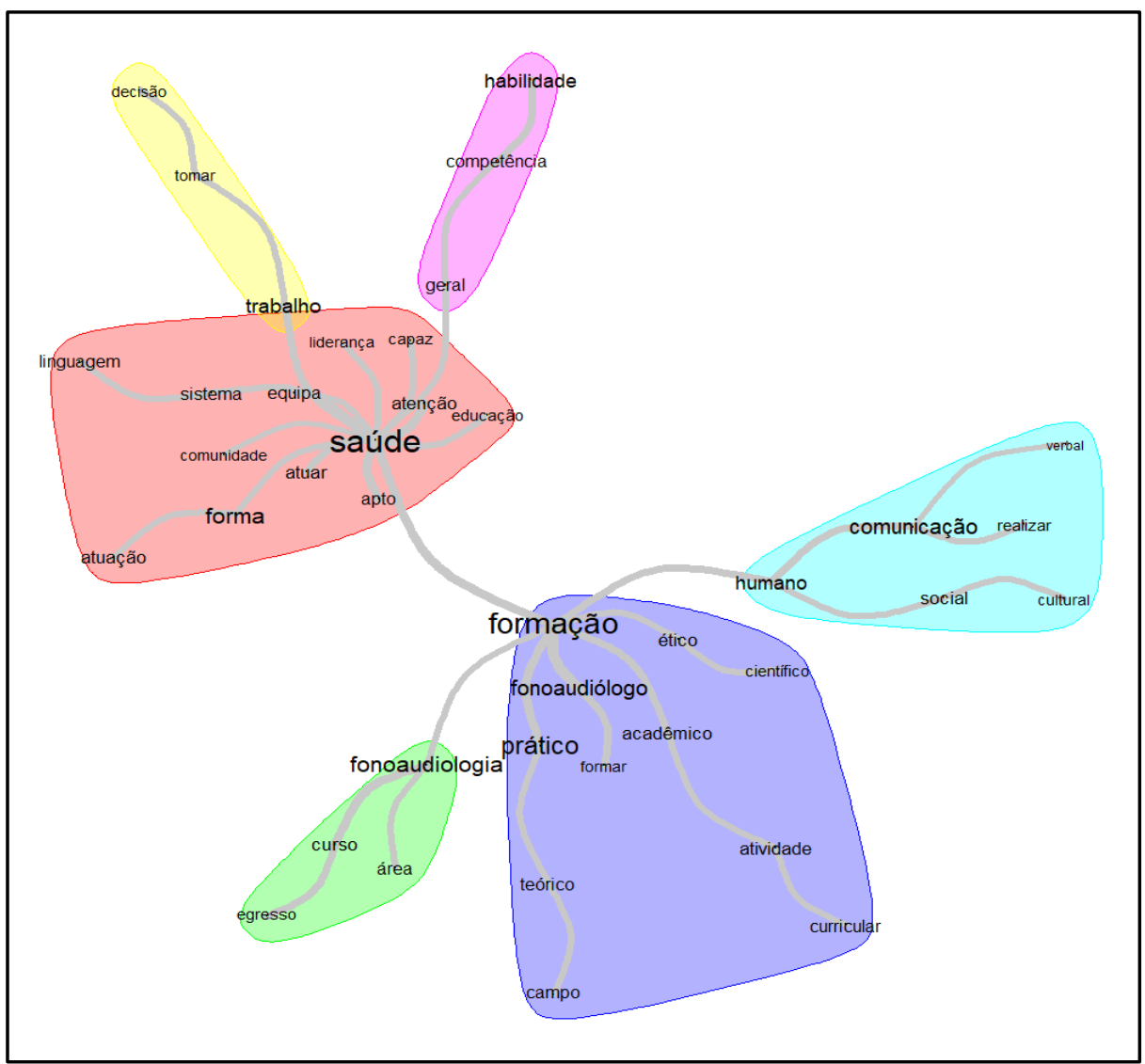

Fonte: Elaborada pelos autores (2020).

O segundo eixo estruturante da Figura 4 vem da palavra "formação". A partir dela compreendemos que o PE das IESs do RS, de modo geral, propõe "formar" "fonoaudiólogos" para a "prática da "fonoaudiologia" e para a "comunicação" "humana". Neste ponto, podemos perceber claramente o distanciamento entre a proposta das DCNs dos PEs, fato que se reflete em outros espaços do país e apontam para um distanciamento do processo ensino-serviço (CORREIA; TELLES; ARAÚJO, 2018; LEMOS; BAZZO, 2010), bem como a necessidade de articular, planejar e construir novas práticas que contemplem tais qualificações (GARCIA, 2015) por meio de reformulações curriculares (TELLES; ARCE, 2015) que saiam do modelo tradicional, focado principalmente em aspectos técnicos da profissão, ou, em outras palavras, no foco central da comunicação humana (SOLEMAN; MARTINS, 2015).

Ao fim desta discussão cabe salientar que a palavra "social", com frequência de $n=7$ nas DCNs e n=14 nos PEs, foi inicialmente pesquisada por fazer parte da expressão 
“comunicação social”. Porém, em todas as suas possibilidades de registro, não apresentou nenhuma ligação semântica com a temática do estudo, sendo propositalmente excluída de toda a análise, afim de não comprometer a qualidade da visualização dos vocábulos nas imagens. Além disso, não houve nenhum impacto nos resultados obtidos por este critério de exclusão.

\section{CONCLUSÃO}

Com este estudo foi possível identificar, através da análise documental das DCNs e dos PEs das IESs do RS, que a abordagem aos conceitos relacionados à comunicação necessita de reformulações, com reflexões mais aprofundadas, buscando construções coletivas e compartilhadas com profissionais da comunicação social (Comunicólogos), considerando a interdisciplina e o grau de importância desta documentação orientadora do processo de formação de profissionais da área da comunicação humana (Fonoaudiólogos).

Observou-se, também, a polissemia do termo "comunicação" e foi possível classificálo para melhor estabelecer suas relações, facilitando assim sua análise. A qualidade multívoca do vocábulo despertou diferentes reflexões e abordagens por múltiplas áreas do conhecimento, cada sentido abordado evoca a necessidade de novas pesquisas para compreender e correlacionar tais conceitos à formação dos profissionais da saúde.

Apenas uma IES fez menção à aplicação da comunicação como instrumento de cuidado e ao fortalecimento do trabalho multiprofissional, o que indica a urgência em rever os PPCs para que possam se adequar, tanto ao perfil esperado para a inserção no sistema de saúde do país quanto para formação de um profissional capaz de atender o sujeito em sua integralidade.

Demonstrou-se também que uma das IESs não faz menção ao termo "comunicação" em seu PE, o que leva ao importante questionamento quanto ao objetivo de uma IES pública, formadora de capital humano para uma profissão de suma importância na habilitação e reabilitação do cidadão brasileiro, que poderá por ventura ser atendido por um profissional tecnicamente incompetente em comunicação social. 
Por fim, o estudo apontou a discrepância conceitual da DCN, que entende a comunicação como habilidade, desconsiderando o processo de domínio do conhecimento visando à modificação de atitudes, em relação aos PEs das IESs do RS que, de modo geral, abordam o vocábulo prioritariamente vinculado à expressão "comunicação humana", reduzindo o perfil do egresso e sua relação com a comunicação a um viés tecnicista.

\section{REFERÊNCIAS}

AGUIAR, A. C.; KALIL, I. R.; SILVEIRA, M.S. M.; BORGES, W.C.; MOTTA, P.H.F.M.; BORGES, G.C. O ensino da comunicação na formação profissional em saúde no Brasil: análise da literatura especializada posterior à homologação das diretrizes curriculares nacionais. Cadernos ABEM, [s. l.], v. 10, p. 69-77, 2014. Disponível em: http://www.ufrgs.br/pediatria/Repositorio/seminarios/subsidios-bibliograficos/documentos-

basicos/Aguiar_O_ensino_da_comunicacao_na_formacao_profissional_em_saude_no_Brasil_CadernosABEM_ 2014.pdf. Acesso em: 18 jun. 2020.

BACHMANN, C.; ABRAMOVITCH, H.; BARBU, C.G.; CAVACO, A.M.; ELORZA, R.D.; HAAK, R.; LOUREIRO, E.; RATAJSKA, A.; SILVER-MAN, J.; WINTERBURN, S.; ROSENBAUM, M. A European consensus on learning objectives for a core communication curriculum in health care professions. Patient Education and Counseling, [s. l.], v. 93, n. 1, p. 18-26, 2013. Disponível em: https://doi.org/10.1016/j.pec.2012.10.016. Acesso em: 10 jul. 2020.

BOISSY, A.; WINDOVER, A.; BOKAR, D.; KARAFA, M.; NEUENDORF, K.; FRANKEL, R.; MERLINO, J.; ROTHBERG, M. Communication skills training for physicians improves patient satisfaction. Journal of General Internal Medicine, [s. l.], v. 31, n. 7, p. 755-761, 2016. Disponível em: https://pubmed.ncbi.nlm.nih.gov/26921153/. Acesso em: 10 fev. 2020.

BOMFIM, R.A. Competência profissional: uma revisão bibliográfica. Revista Organização Sistêmica, [s. l.], v. 1, n. $1, \quad$ p. $1-18, \quad 2012 . \quad$ Disponível em: https://www.uninter.com/revistaorganizacaosistemica/index.php/organizacaoSistemica/article/view/62/36. Acesso em: 10 abr. 2020.

BRAGA, J. L.; CALAZANS, R. Comunicação e educação: questões delicadas na interface. São Paulo: Hacker, 2001.

BRASIL. Resolução CNE/CES n. 5, de 19 de fevereiro de 2002. Institui Diretrizes Curriculares Nacionais do Curso de Graduação em Fonoaudiologia. Diário Oficial da União: seção 1, Brasília, DF, p. 12, 2002. Disponível em: http://portal.mec.gov.br/cne/arquivos/pdf/CES052002.pdf. Acesso em: 17 mar. 2020.

BURGOS, M.S.; ETGES, G.M.; SILVA, P.T.; ROCHA, D.; AGUIAR, S. Interdisciplinaridade e integração pesquisa, ensino e extensão na promoção da saúde: a comunicação social na saúde da comunidade e educação básica. Cinergis, [s. l.], v. 16, n. 4, p. 283-291, 2015. Disponível em: https://doi.org/10.17058/cinergis.v16i4.6939. Acesso em: 18 jun. 2020.

CÂMARA, R.H. Análise de conteúdo: da teoria à prática em pesquisas sociais aplicadas às organizações. Gerais: Revista Interinstitucional de Psicologia, Belo Horizonte, v. 6, n. 2, p. 179-191, 2013. Disponível em: http://pepsic.bvsalud.org/pdf/gerais/v6n2/v6n2a03.pdf. Acesso em: 5 mar. 2020.

CAMARGO, B.V.; JUSTO, A.M. IRAMUTEQ: um software gratuito para análise de dados textuais. Temas em Psicologia, Ribeirão Preto, v. 21, n. 2, p. 513-518, 2013. Disponível em: https://doi.org/10.9788/TP2013.2-16. Acesso em: 5 mar. 2020. 
CAPRARA, A.; FRANCO, A.L.S. The patient-physician relationship: towards humanization of medical practice. Cadernos de Saúde Pública, Rio de Janeiro, v. 15, n. 3, p. 647-654, 1999. Disponível em: https://doi.org/10.1590/s0102-311X1999000300023. Acesso em: 23 maio 2020.

CECCIM, R.B.; FEUERWERKER, L.C.M. Mudança na graduação das profissões de saúde sob o eixo da integralidade. Cadernos de Saúde Pública, Rio de Janeiro, v. 20, p. 1400-1410, 2004. Disponível em: https://doi.org/10.1590/S0102-311X2004000500036. Acesso em: 13 jan. 2020.

CORREIA, T.M.; TELLES, M.W.P.; ARAÚJO, M.V.R. A formação em saúde coletiva na visão de estudantes de Graduação em fonoaudiologia da UFBA. Distúrbios da Comunicação, São Paulo, v. 30, n. 4, p. 679-687, 2018. Disponível em: https://doi.org/10.23925/2176-2724.2018v30i4p679-687. Acesso em: 15 mar. 2020.

COSTA, A.R.C.; BENTO, A.V. Práticas e comportamentos de liderança na gestão dos recursos humanos escolares. Ensaio: Avaliação e Políticas Públicas em Educação, Rio de Janeiro, v. 23, n. 88, p. 663-680, 2015. Disponível em: http://www.scielo.br/scielo.php?script=sci_arttext\&pid=S0104-40362015000300663\&lng=pt\&nrm=iso. Acesso em: 15 mar. 2020.

DONATO, A.F.; GOMES, A.L.Z. O estudo da comunicação na formação dos profissionais de saúde: algumas questões e aproximações. Boletim do Instituto de Saúde, São Paulo, v. 12, n. 1, p. 37-43, 2010. Disponível em: http://periodicos.ses.sp.bvs.br/scielo.php?script=sci_arttext\&pid=S1518-

18122010000100007\&lng=pt\&nrm=iso. Acesso em: 18 jun. 2020.

DROESCHER, F.D.; SILVA, E.L. O pesquisador e a produção científica. Perspectivas em Ciência da Informação, Belo Horizonte, v. 19, n. 1, p. 170-189, 2018. Disponível em: https://telematicafractal.com.br/revista/index.php/telfract/article/view/7. Acesso em: 18 jun. 2020.

ELLIS, W.R.; DIETZ, W.H. A new framework for addressing adverse childhood and community experiences: the building community resilience model. Academic Pediatrics, [s. l.], v. 17, n. 7S, p. S86-S93, 2017. Disponível em: https://www.academicpedsjnl.net/action/showPdf?pii=S1876-2859\%2816\%2930552-6. Acesso em: 15 jun. 2020.

FERIGOLLO, J.P.; KESSLER, T.M. Physiotherapy, speech, language and hearing sciences and occupational therapy: interdisciplinary practice in disorders of human communication. Revista CEFAC, São Paulo, v. 19, n. 2, p. 147158, 2017. Disponível em: https://doi.org/10.1590/1982-0216201719213816. Acesso em: 15 jun. 2020.

FERMINO, T.Z.; CARVALHO, E.C. A comunicação terapêutica com pacientes em transplante de medula óssea: perfil do comportamento verbal e efeito de estratégia educativa. Cogitare Enfermagem, Curitiba, v. 12, n. 3, p. 287-295, 2007. Disponível em: https://revistas.ufpr.br/cogitare/article/view/10022/6883. Acesso em: 3 mar. 2020.

FERREIRA, M.A. A comunicação no cuidado: uma questão fundamental na enfermagem. Revista Brasileira de Enfermagem, Brasília, v. 59, n. 3, p. 327-330, 2006. Disponível em: https://doi.org/10.1590/s003471672006000300014. Acesso em: 3 mar. 2020.

FLEURY, M.T.L.; FLEURY, A. Construindo o conceito de competência. Revista de Administração Contemporânea, Curitiba, v. 5, p. 183-196, 2001. Disponível em: https://doi.org/10.1590/S141565552001000500010. Acesso em: 20 abr. 2020.

FRANCO, C.A.G.S.; CUBAS, M.R.; FRANCO, R.S. Currículo de medicina e as competências propostas pelas diretrizes curriculares. Revista Brasileira de Educação Médica, Rio de Janeiro, v. 38, n. 2, p. 221-230, 2014. Disponível em: https://doi.org/10.1590/S0100-55022014000200009. Acesso em: 10 mar. 2020.

FREY, K. Desenvolvimento sustentável local na sociedade em rede: o potencial das novas tecnologias de informação e comunicação. Revista de Sociologia e Política, Curitiba, n. 21, p. 165-185, 2003. Disponível em: https://doi.org/10.1590/S0104-44782003000200011. Acesso em: 7 fev. 2020. 
GARCIA, V.L. Formação do Fonoaudiólogo e sua atuação na área educacional. In: QUEIROGA, B.A.M.; ZORZI, J.L.; GARCIA, V.L. (org.). Fonoaudiologia educacional: reflexões e relatos de experiências. Brasília, DF: Kiron, 2015. p. 54-66.

KITSON, A.; MARSHALL, A.; BASSETT, K.; ZEITZ, K. What are the core elements of patient-centred care? A narrative review and synthesis of the literature from health policy, medicine and nursing. Journal of Advanced Nursing, [s. l.], v. 69, n. 1, p. 4-15, 2013. Disponível em: https://doi.org/10.1111/j.1365-2648.2012.06064.x. Acesso em: 27 maio 2020.

KURTZ, S.; COOKE, L. Learner-centred communication training. In: KISSANE, D.; BULTZ, B.; BUTOW, P.; FINLAY, I. Handbook of communication in oncology and palliative care. New York: Oxford University Press, 2017. p. 61-70.

LEMOS, M. A integração ensino-serviço no contexto da formação do fonoaudiólogo: um relato de experiência da prática de ensino-aprendizagem no estágio de saúde coletiva. Revista Baiana de Saúde Pública, Salvador, v. 36, n. 4, p. 1068-1076, 2012. Disponível em: http://files.bvs.br/upload/s/0100-0233/2013/v36n4/a3797.pdf. Acesso em: 10 jun. 2020.

LEMOS, M.; BAZZO, L.M.F. Formação do fonoaudiólogo no município de Salvador e consolidação do SUS. Ciência \& Saúde Coletiva, Rio de Janeiro, v. 15, n. 5, p. 2563-2568, 2010. Disponível em: https://doi.org/10.1590/S141381232010000500030 . Acesso em: 10 jun. 2020.

MARCHAND, P.; RATINAUD, P. L'analyse de similitude appliquée aux corpus textuels: les primaires socialistes pour l'élection présidentielle française (septembre-octobre 2011). In: JOURNEES INTERNATIONALES D'ANALYSE STATISTIQUE DES DONNEES TEXTUELLES, 2011, Paris. Anais... Paris: [s. n.], 2011. p. 687-699. Disponível em: http://lexicometrica.univ-paris3.fr/jadt/jadt2012/Communications/Marchand,\%20Pascal\%20et\%20al.\%20\%20L'analyse\%20de\%20similitude\%20appliquee\%20aux\%20corpus\%20textuels.pdf. Acesso em: 5 fev. 2020.

MULLAN, B.A.; KOTHE, E.J. Evaluating a nursing communication skills training course: The relationships between self-rated ability, satisfaction, and actual performance. Nurse Education in Practice, [s. l.], v. 10, n. 6, p. 374-378, 2010. Disponível em: https://doi.org/10.1016/j.nepr.2010.05.007. Acesso em: 16 abr. 2020.

NESTEL, D.; MUIR, E.; PLANT, M.; KIDD, J.; THURLOW, S. Modelling the lay expert for first-year medical students: the actor-patient as teacher. Medical Teacher, [s. l.], v. 24, n. 5, p. 562-564, 2002. Disponível em: https://pubmed.ncbi.nlm.nih.gov/12450482/. Acesso em: 16 abr. 2020.

PEREIRA, A.L.F. As tendências pedagógicas e a prática educativa nas ciências da saúde. Cadernos de Saúde Pública, Rio de Janeiro, v. 19, n. 5, p. 1527-1534, 2003. Disponível em: https://doi.org/10.1590/S0102311X2003000500031. Acesso em: 16 abr. 2020.

PEREIRA, D.M.; SILVA, G.S. As Tecnologias de Informação e Comunicação (TICs) como aliadas para o desenvolvimento. Cadernos de Ciências Sociais Aplicadas, [s. l.], n. 8, p. 151-174, 2011. Disponível em: http://periodicos2.uesb.br/index.php/ccsa/article/view/1935. Acesso em: 7 jul. 2020.

PINHEIRO, F.M.F.M.; SANTOS, O.C.; SILVA, J.C.B.; BARBOSA, L.A.L.; CARVALHO, L.V.; MOTA, S.M. A. O perfil de liderança dos enfermeiros na Atenção Básica à Saúde. Revista Eletrônica Acervo Saúde, [s. l.], n. 43, p. e2793e2793, 2020. Disponível em: https://doi.org/10.25248/reas.e2793.2020. Acesso em: 7 jul. 2020.

RANJAN, P.; KUMARI, A.; CHAKRAWARTY, A. How can doctors improve their communication skills? Journal of Clinical and Diagnostic Research, [s. l.], v. 9, n. 3, p. JE01-JE04, 2015. Disponível em: https://www.ncbi.nlm.nih.gov/pmc/articles/PMC4413084/pdf/jcdr-9-JE01.pdf. Acesso em: 10 mar. 2020.

ROCHA, C.M.V. Comunicação social e vacinação. História, Ciências, Saúde-Manguinhos, Rio de Janeiro, v. 10, p. 795-806, 2003. Disponível em: https://doi.org/10.1590/S0104-59702003000500017. Acesso em: 7 mar. 2020.

RODRIGUES, C.; BLATTMANN, U. Gestão da informação e a importância do uso de fontes de informação para geração de conhecimento. Perspectivas em Ciência da Informação, [s. l.], v. 19, n. 3, p. 4-29, 2014. Disponível em: https://doi.org/10.1590/1981-5344/1515. Acesso em: 10 mar. 2020. 
ROSSI, P.S.; BATISTA, N.A. O ensino da comunicação na graduação em medicina: uma abordagem. Interface Comunicação, Saúde, Educação, Botucatu, v. 10, n. 19, p. 93-102, 2006. Disponível em: https://doi.org/10.1590/S1414-32832006000100007. Acesso em: 20 jan. 2020.

SAUPE, R.; BENITO, G.A.V.; WENDHAUSEN, Á.L.P.; CUTOLO, L.R.A. Conceito de competência: validação por profissionais de saúde. Saúde em Revista, Piracicaba, v. 8, n. 18, p. 31-37, 2006. Disponível em: http://189.28.128.100/nutricao/docs/Enpacs/pesquisaArtigos/conceito_de_competencia_validacao_por_prof _saude_2006.pdf. Acesso em: 20 jan. 2020.

SOLEMAN, C.; MARTINS, C.L. O trabalho do fonoaudiólogo no Núcleo de Apoio à Saúde da Família (NASF): especificidades do trabalho em equipe na atenção básica. Revista CEFAC, São Paulo, v. 17, n. 4, p. 1241-1253, 2015. Disponível em: https://doi.org/10.1590/1982-0216201517417114. Acesso em: 1 jun. 2020.

TABORDA, M.G. A atividade de Comunicação Social e a formação da opinião pública. Revista de Direito Econômico e Socioambiental, Curitiba, v. 8, n. 3, p. 410-432, 2017. Disponível em: https://doi.org/10.7213/rev.dir.econ.soc.v8i3.18897. Acesso em: 1 jun. 2020.

TEIXEIRA, R.R. Models of communication and health practices. Interface - Comunicação, Saúde, Educação, Botucatu, v. 1, n. 1, p. 7-40, 1997. Disponível em: https://doi.org/10.1590/S1414-32831997000200002. Acesso em: 18 maio 2020.

TELLES, M.W.P; ARCE, V.A.R. Training and PET-Saúde: speech, hearing and language students' experiences in Bahia. Revista CEFAC, São Paulo, v. 17, n. 3, p. 695-706, 2015. Disponível em: https://doi.org/10.1590/1982021620159514. Acesso em: 5 maio 2020.

\section{(cc) $\mathrm{BY}$}

Este trabalho está licenciado com uma Licença Creative Commons - Atribuição 4.0 Internacional. 